UCRL-JC-128441

PREPRINT

\title{
Scaling Behavior in Interference Lithography
}

R. R. Agayan, W. C. Banyai, A. Fernandez

This paper was prepared for submittal to the 23rd Annual International Symposium on Microlithography Emerging Lithographic Technologies II Santa Clara, CA

February 22-27, 1998

February 27, 1998

This is a preprint of a paper intended for publication in a journal or proceedings. Since changes may be made before publication, this preprint is made available with the understanding that it will not be cited or reproduced without the permission of the author. 


\section{DISCLAIMER}

This document was prepared as an account of work sponsored by an agency of the United States Government. Neither the United States Government nor the University of California nor any of their employees, makes any warranty, express or implied, or assumes any legal liability or responsibility for the accuracy, completeness, or usefulness of any information, apparatus, product, or process

disclosed, or represents that its use would not infringe privately owned rights. Reference herein to any specific commercial product, process, or service by trade name, trademark, manufacturer, or otherwise, does not necessarily constitute or imply its endorsement, recommendation, or favoring by the United States Government or the University of California. The views and opinions of authors expressed herein do not necessarily state or reflect those of the United States Government or the University of California, and shall not be used for advertising or product endorsement purposes. 


\title{
Scaling behavior in interference lithography
}

\author{
Rodney R. Agayan, William C. Banyai, Andres Fernandez \\ Lawrence Livernore National Laboratory, Livermore, CA 94550
}

\begin{abstract}
Interference lithography is an emerging technology that provides a means for achieving high resolution over large exposure areas $\left(-1 \mathrm{~m}^{2}\right)$ with virtually unlimited depth of ficld. Onc- and two-dimensional arrays of deep submicron structures can be created using near i-line wavelengths and standard resist processing. In this paper, we report on recent advances in the devclopment of this technology, focusing, in particular, on how exposure latitude and resist profile scale with interference period We present structure width vs dose curves for periods ranging from $200 \mathrm{~nm}$ to $10 \mu \mathrm{m}$, demonstrating that deep submicron structures can be generated with exposure latitudes exceeding $30 \%$. Our experimental results are compared to simulations based on PROLITH/2.
\end{abstract}

Keywords. interference lithography, scaling, submicron, freeze-drying, exposure latitude, PROLITH/2, Lumped Parameter Model

\section{INTRODUCTION}

The increasing demands of the flat panel display (FPD) industry towards smaller critical dimension (CD) structures over large field sizes pose marked challenges for current microlithographic technologies. The prospect of field-emission display (FED) production as a viable FPD technology hinges on the lithographic ability to fabricate high-density emitter-tip or hole arrays to improve display brightness, reduce power consumption and increase display lifetime. Targeted CDs are in the range of $05-1 \mu \mathrm{m}$, about five times smaller than those achieved on active matrix liquid crystal displays (AMLCDs), the currently dominant technology.' Other lithographic technologies such as e-beam lithography or X-ray lithography have the submicron resolution capabilities, but they have difficulties meeting the large-field and high-volume requirements of FED manufacture in a simple, cost-effective manner.

Conventional optical lithography, which is currently used for AMLCD fabrication, has several inherent problerns limiting its large area, high-resolution performance It is well known that resolution in conventional lithography is diffraction limited. To improve resolution, which is given by $k \lambda N \mathrm{NA}$, where $k$ is a factor depending on the resist processing technology and NA is the lens numerical aperture, the source wavelength $\lambda$ must be reduced or NA of the imaging system increased Both of these options, however, have the effect of decreasing the depth of focus $\left(-\lambda N \mathrm{NA}^{2}\right)$. To obtain high-resolution, substrate positioning must be precisely controlled and critical mechanical components may be required. The imaging system also limits the workable field size; thus, step-and-repeat techniques arc often employed to pattern large areas. These procedures can be time-consuming and costly, and they often introduce intolerable stitching errors. The development of a cost-effective technology that can deliver both increased resolution and field size remains a significant challenge.

Interference lithography (IL) is a maskless technology that may be able to meet this challenge. In IL, resist coated substrates are exposed to an intensity distribution produced by interfering multiple, mutually coherent wavefronts. This interference pattern exists within the volume of space defined by the overlapping beams and is invariant normal to the substrate; thus, the depth of focus is essentially infinite in comparison to typical semiconductor device topographies. Because IL does not require a mask, field size only depends on the size of the beams. Single, large-area exposures can be implemented, eliminating the possibility of stitching errors. The other principal advantage of IL is that resolution is not limited by diffraction. Instead, the aerial image is a sinusoidal intensity distribution that can have periodicity as small as half the source wavelength. Using an i-line source, for a 1:1 (line : space) ratio, linewidths as small as $100 \mathrm{~nm}$ can be achieved, whereas modern day optical steppers need phase-shift mask and off-axis illumination techniques Large-area, 100-nm period gratings

Further author information -

R.R.A (correspondence): Email: agayan $1 @$ llnl gov; Telephone: 510-422-5300; Fax: 510-423-1488

W.C.B : Email: banyail @llnl.gov; Telephone 510-424-3536, Fax: 510-423-1488

A.F : Email: fernandez10@linl gov; Tclephone: 510-424-2904; Fax: 510-423-1488 
and grids have been fabricated using achromatic IL techniques. ${ }^{2}$ The intrinsic simplicity that makes IL a relatively inexpensive technology also introduces its own drawback; images are restricted to simple, periodic patterns. However, for applications in which repetitive patterns suffice, $\mathrm{IL}$ is an attractive alternative to conventional methods.

Having the capability of patterning submicron, high aspect ratio periodic structures with a virtually unlimited depth-of-field, IL is a robust technology whose utility is gradually gaining recognition. In recent years, IL has been used in a wide variety of applications. One-dimensional (1-D) structures have been generated with IL to create highly efficient metallic ${ }^{3}$ and dielectric $^{4}$ diffraction gratings, $x$-ray transmission gratings, ${ }^{5}$ and extreme-ultraviolet polarizers. ${ }^{6}$ Two-dimensional (2-D) arrays of posts or holes can also be fabricated using double-exposure, ${ }^{7-9}$ multiple-beam $(>2),{ }^{9,10}$ or image reversal ${ }^{10,11}$ techniques. Because it can accommodate the volume manufacture of these high-resolution, 2-D patterns over large areas, IL is a technology of great interest to the FED industry, with production-level systems currently being developed. ${ }^{12}$ These 2-D structures also have potential applications for magnetic high-density storage devices. ${ }^{13}$ By using multiple exposures with variations in period, phase, and orientation, more complex 1-D and 2-D structures resembling moiré patterns can be achieved. $^{14}$

The commercial viability of IL will depend ultimately on such attributes as uniformity, repeatability and process control. In this article, we investigate these factors, concentrating primarily on exposure latitude and its dependence on interference period. It is expected that exposure latitude in IL will remain constant for decreasing periods. Previous efforts to fabricate structures at a $300-\mathrm{nm}$ pitch have been successful, and we extend those efforts, showing that high-aspect ratio, vertical sidewall structures can be created with a high degree of exposure latitude at a $200-\mathrm{nm}$ pitch. We compare and contrast computer simulated CD vs. exposure dose curves to our experimental data, and we explain how exposure latitude scales with decreasing periods using the model theory. We then discuss the practical significance of this scaling and describe other contributing effects not modeled by the simulations.

\section{APPARATUS}

The exposure system consists of a simple interferometer operating at a wavelength of $351.1 \mathrm{~nm}$, as shown in Figure 1 . To control the period, $\Lambda$, of the interference pattern, the half-angle $\theta$ between both arms is adjusted according to the grating equation, $\Lambda=\lambda /(2 \sin \theta)$. Thus, for gratings with $\Lambda=1.0 \mu \mathrm{m}$ and $200 \mathrm{~nm}, \theta$ is set to $10.1^{\circ}$ and $61.4^{\circ}$, respectively. Resistcoated silicon substrates are held firmly in the exposure plane on a vacuum chuck. Since the interference pattern is generated within the volume of intersection of the two arms, substrate positioning is not a critical issue. However, the normal of the substrate is aligned to bisect the two incoming beams to within $1 / 2$ degree so that the fringes on the substrate are not tilted. In

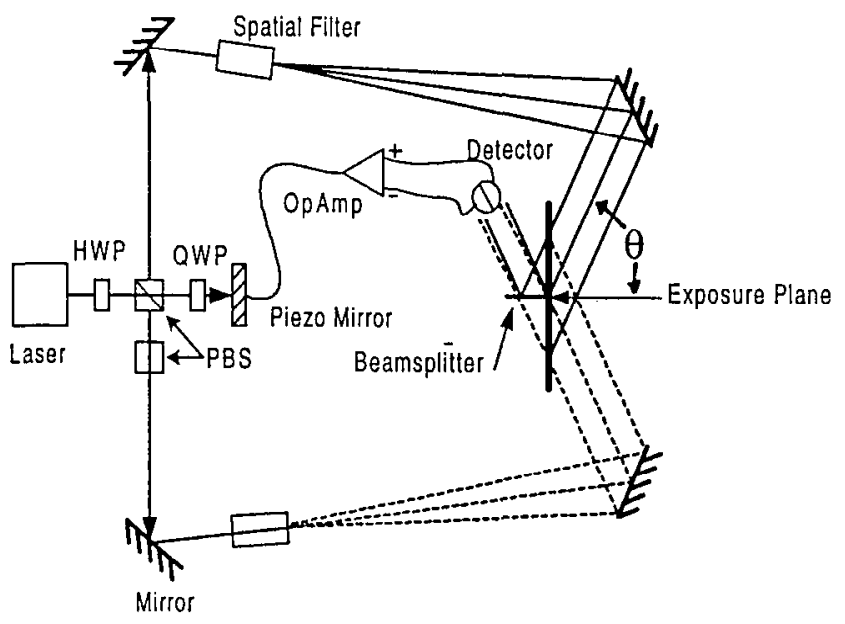

Figure 1: Interference lithography exposure system (HWP: half-wave plate; QWP: quarter-wave plate; PBS: polarizing beamsplitter) 
order to realize high-quality interference fringes, the design of the system, which differs from our previously used systems, ${ }^{7}$ addresses several issues:

1) polarization extinction ratio of each arm;

2) fringe visibility;

3) intensity variation across the wafer diameter;

4) phase error across the beam; and

5) relative phase stability between the two beams.

A subsystem, depicted in Figure 2, allows control of the relative intensity between the two beams while maintaining a polarization extinction ratio in each arm of 100:1, with both arms adjusted to be s-polarized. The light exiting the laser is spolarized with an extinction ratio of 100:1. This light first passes through a half-wave plate (HWP) before passing through a polarization beamsplitter (PBS). The HWP is adjusted such that light impinging on the PBS has equal parts s-and p-polarized light The PBS has the characteristic that p-polarized light is transmitted with 95\% efficiency and polarized 100.1. The transmitted light passes through a quarter-wave plate (QWP), reflects off a normal incidence mirror, passes back through the QWP, and is reflected by the PBS since the light is now s-polarized. The PBS has the characteristic that almost $100 \%$ of incident s-polarized light and 5\% of incident p-polarized light is reflected. So, while the arm formed from light passing through the QWP twice is polarized 100.1, the light directed from the laser that is reflected in the opposite direction is only polarized 20.1 . In order to alleviate this problem, a second PBS, rotated $90^{\circ}$ to transmit s-polarized light, is placed in this arm to bring the polarization extinction ratio to $100: 1$.

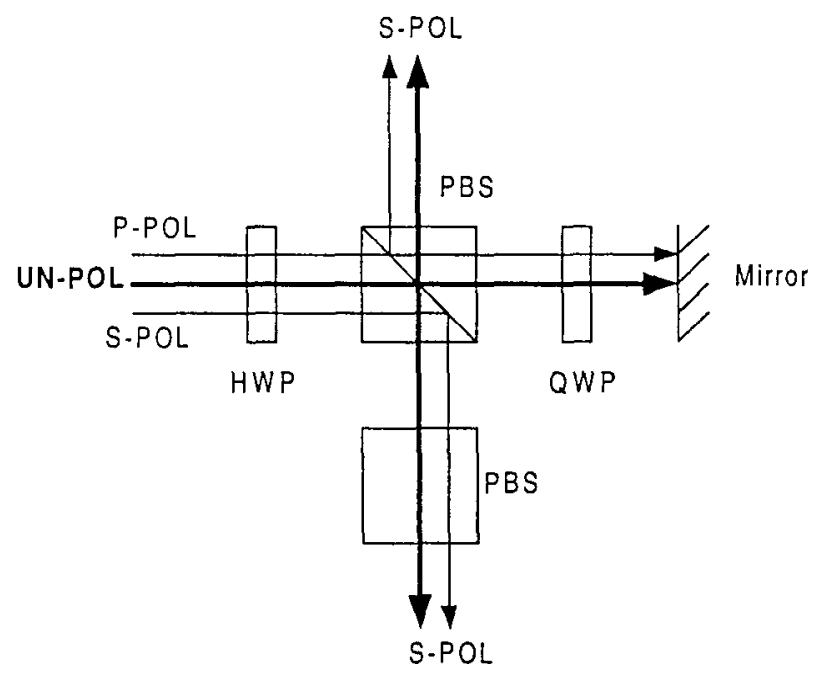

Figure 2: Subsystem for splitting incoming beam into two S-polarized beams and controlling the relative intensities. (UN-POL: unpolarized; P-POL: parallel polarized; S-POL: perpendicularly polarized; PBS: polarizing beamsplitter; HWP: halfwave plate; QWP: quarter-wave plate)

The fringe visibility of the system is determined by many factors including the relative power in each arm, the temporal and spatial coherence of the laser, and the relative path length difference between the two arms. The power in each arm is measured with a calibrated photodiode power meter and the HWP is adjusted until the powers are equal to within $1 \%$. A Coherent Innova Sabre ion laser operating in a single-frequency $\mathrm{TEM}_{\infty}$ mode is used with an estimated coherence length greater than $1 \mathrm{~m}$. The path length difference between the two arms is adjusted to be within $2 \mathrm{~cm}$. Therefore, the fringe visibility is maximized.

In order to achieve large area, uniform exposures, two conditions are met for each beam: 1) spatial filtering and 2) beam expansion such that the intensity at the exposure plane at a radius of 1.5 inches is not less than $90 \%$ of the center intensity. The system does not have collimation optics after the spatial filters; instead, the beams are allowed to expand over a distance 
of $3.5 \mathrm{~m}$ after spatial filtering causing a small cumulative phase error of 1.5 waves from center to a radius of 1.5 inches. A small amount of additional wavefront error, 1/10 wave RMS, is added by the 6-inch diameter turning mirrors.

The laser is specified to have a virtual beam waist of $0.8 \mathrm{~mm}$ at a distance of $8.5 \mathrm{~m}$ behind the output coupler. The distance from the output coupler to the spatial filter is $2 \mathrm{~m}$ and from the spatial filter to the exposure plane is $3.5 \mathrm{~m}$. Propagating the Gaussian beam in terms of the complex propagation constant using ABCD matrices, one finds that in order to meet condition (2), the beam waist at the spatial filter must be $2.2 \mu \mathrm{m}$. Using the criterion that a pinhole aperture of $4.6 \times$ beam waist minimizes the intensity ripple in the expanding beam to $1 \%$, the pinhole diameter must be $10 \mu \mathrm{m}$. The focal length of the spatial filter microscope objective is calculated using the relation $\omega_{i} \omega_{o}=f \lambda \pi$, where $\omega_{i}$ is the input beam waist, $\omega_{0}$ is the beam waist at focus, $f$ is the focal length, and $\lambda$ is the wavelength. The input beam waist is calculated by propagating the beam from the virtual beam waist of the laser cavity to the spatial filter, a distance of $4.5 \mathrm{~m}$, yielding an input beam waist of $1 \mathrm{~mm}$. As a result, the focal length of the microscope objective is $20 \mathrm{~mm}$.

Since differential phase noise between the two beams causes the fringe position to vary with time, effectively washing out the fringes, vibration damping and active fringe stabilization are required. The main sources of noise in our system were vibration-induced index changes in the air through which the beams propagated and vibrations coupled to the optics mounts. To suppress floor and air vibrations, the table is pneumatically isolated and the apparatus is enclosed in a plastic case during cxposurcs. Because this is not enough to eliminate all noise, a fringe stabilization system is employed. Portions of the interfering beams are recombined, as shown in Figure 1, to produce fringes with a spatial frequency of approximately $1 \mathrm{~cm}^{-1}$, which are differentially detected by an Odhner Holographics Stabilock II Active Fringe Stabilizer. The feedback signal is applied to a piezo-actuated mirror, as shown in Figure 1. The system has a frequency response up to $2.5 \mathrm{kHz}$.

\section{FABRICATION}

Standard processing techniques are used to generate $1.0-\mu \mathrm{m}$ period structures. Silicon wafers are first spin coated with $\sim 150 \mathrm{~nm}$ of Brewer Science XHRi-16 anti-reflective coating (ARC) and then baked at $185^{\circ} \mathrm{C}$ for 15 minutes in a conventional oven. They are immediately spin coated with $\sim 670 \mathrm{~nm}$ of Shipley $507-\mathrm{A}$ resist and soft baked at $90^{\circ} \mathrm{C}$ for 30 minutes. The resist-coated substrates are then exposed to the interference pattern. A detector, oriented normal to the incident beams, measures the power just before each exposure. The applied dose is varied by changing the exposure time for a set power. After exposure, the samples are developed for 60 seconds in Shipley MF-701 (a $0.237 \mathrm{~N}$ tetramethylammonium hydroxide) developer, rinsed in de-ionized water and dried with dry nitrogen.

We found that fabrication of structures with periods less than $300 \mathrm{~nm}$ is complicated by a strong tendency for the patterns to collapse during the rinse process. ${ }^{15,16}$ This problem has been observed before and is caused by the large surface tension of the rinse liquid. Techniques for prevention of resist pattern collapse fall into three categories (1) using a rinse liquid with decreased surface tension; ${ }^{17}$ (2) improving resist structure integrity by reducing the aspect ratio, controlling resist hardness during the rinse process, ${ }^{18}$ using a resist with a higher Young's modulus, ${ }^{19}$ or optimizing adhesion and wetting properties; ${ }^{20}$ (3) eliminating the presence of a gas-liquid interface during drying by using methods such as critical point drying or sublimation. ${ }^{21}$

To generate 200-nm period structures, we use a combination of the above techniques. We improve grating structure stability by spinning only $250 \mathrm{~nm}$ of a 1:1 (resist : ethyl lactate) dilution of the Shipley photoresist on top of the ARC layer. Because many low surface tension rinse solvents have a tendency to dissolve the resist, we choose to use de-ionized water as the rinse liquid in a freeze-drying process. After development, each sample is immersed in de-ionized water. The sample is then removed and placed in a freezer such that a bead of water continuously covers the top surface of the substrate. After freezing, the ice is sublimated in a vacuum chamber. It is important to make sure that the ice does not melt before the pressure falls below the triple-point of water. This is accomplished by using a frozen, relatively massive heat-conducting support. Before removal from vacuum, the samples are allowed to reheat to room temperature to avoid condensation. This freeze-drying process substantially reduces resist collapse, but because the water expands upon freezing, large-field uniform gratings can not be produced. 

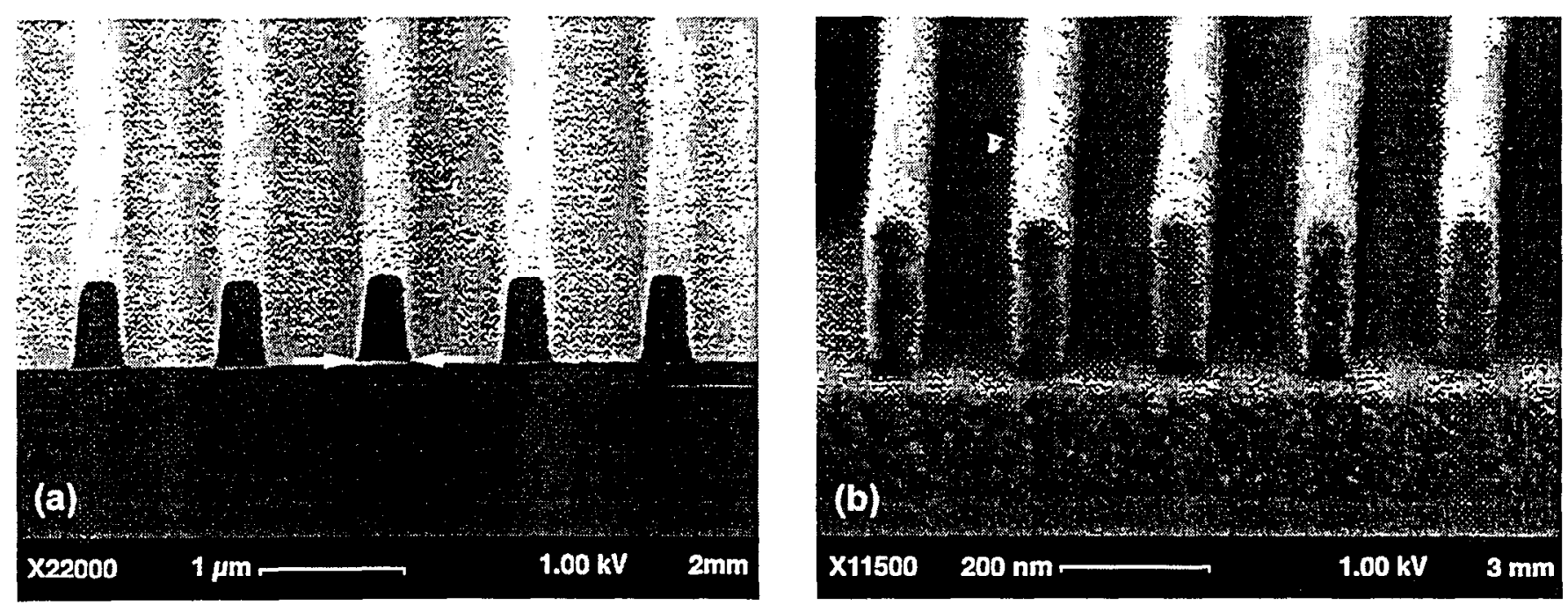

Figure 3: Scanning Electron Micrographs (SEMs) of gratings in Shipley 507-A resist over $150 \mathrm{~nm}$ ARC using a $351.1-\mathrm{nm}$ Ar ion source. (a) 1.0- $\mu \mathrm{m}$ period structures with $18: 1$ aspect ratio, 380-nm linewidth, $84^{\circ}$ sidewall slope (b) $200-\mathrm{nm}$ period structures with $36: 1$ aspect ratio, 70-nm linewidth, vertical sidewalls. The corrugated sidewalls evident at this resolution reveal standing wave effects.

\section{RESULTS AND DISCUSSIONS}

In an effort to assess the viability of IL technology for commercial applications, we have explored process control behavior for sub-micron feature sizes. We present measurements of gratings fabricated at these reduced dimensions Modeling our process using a version of the simulation software PROLITH/2 $2^{22}$ designed for interferometric lithography, we provide explanations and describe the practical significance of the behavior attained.

The scanning electron micrographs (SFMs) of Figures 3(a) and 3(b) show examples of the high-resolution gratings that can be fabricated with IL at a $1.0-\mu \mathrm{m}$ and $200-\mathrm{nm}$ period, respectively. The aspect ratios of these gratings are $1.8 \cdot 1$ (height : width) with a height of $670 \mathrm{~nm}$ for the $1.0-\mu \mathrm{m}$ grating and $3.6: 1$ with a height of $250 \mathrm{~nm}$ for the $200-\mathrm{nm}$ grating. The sidewalls of the $1.0-\mu \mathrm{m}$ period grating are slightly sloped with an angle of $84^{\circ}$ whereas those of the 200 -nm period grating are essentially vertical. This can be expected since the slope of the aerial image increases for decreasing periods. The corrugated sidewalls of the structures in Figure 3(b) reveal the presence of standing waves. Although UV reflection from the silicon substrate is suppressed by the ARC layer, the ARC itself is not perfectly index-matched to the resist, thus some standing wave effects are expected.

The high degree of process control possible with IL is illustrated by Figure 4(a) which displays curves of linewidth, or CD, vs. exposure dose for different grating periods. All CDs are measured at the base of each structure as indicated by the arrows in Figure 3(a). The exposure dose is equal to $2 E_{0}$ where $E_{0}$ is the measured fluence of one beam. Process latitude is roughly proportional to the slope of a smooth curve through the data. Using a linear fit of the data for $\Lambda=1.0 \mu \mathrm{m}$, at a target $\mathrm{CD}$ of $\sim 170 \mathrm{~nm}, \mathrm{a} \pm 10 \%$ change in CD corresponds to an exposure latitude of $\pm 32 \%$. For $\Lambda=200 \mathrm{~nm}$, at a target CD of $\sim 80 \mathrm{~nm}$, a $\pm 10 \%$ change in CD allows a $\pm 34 \%$ change in exposure dose. These measurements are the largest process latitudes reported to date with IL. They demonstrate that, using IL, wide exposure latitudes can be maintained for periods as small as $200 \mathrm{~nm}$.

To investigate how the $C D$ measurements scale with interference period, we have plotted in Figure 4(b) the linewidth, normalized to the period, vs. corrected dose. The method for correcting the dose deserves some explanation. As the interference angle $\theta$ is varied, not only does the period $\Lambda$ change according to the grating equation, but the amplitude of the latent image in the resist changes as well. That is, the fluence absorbed by the resist at the intensity maxima is a function of the interference angle. To first order, this dependence scales as $\cos \theta$ since the beam energy is spread over a larger area of the exposure plane at more oblique angles. Using PROLITH/2, a full calculation of this dependence is performed numerically and is plotted in Figure 5. The plot gives the amplitude correction factor $A(\theta)$ vs. interference angle $\theta$ for two 
(a)

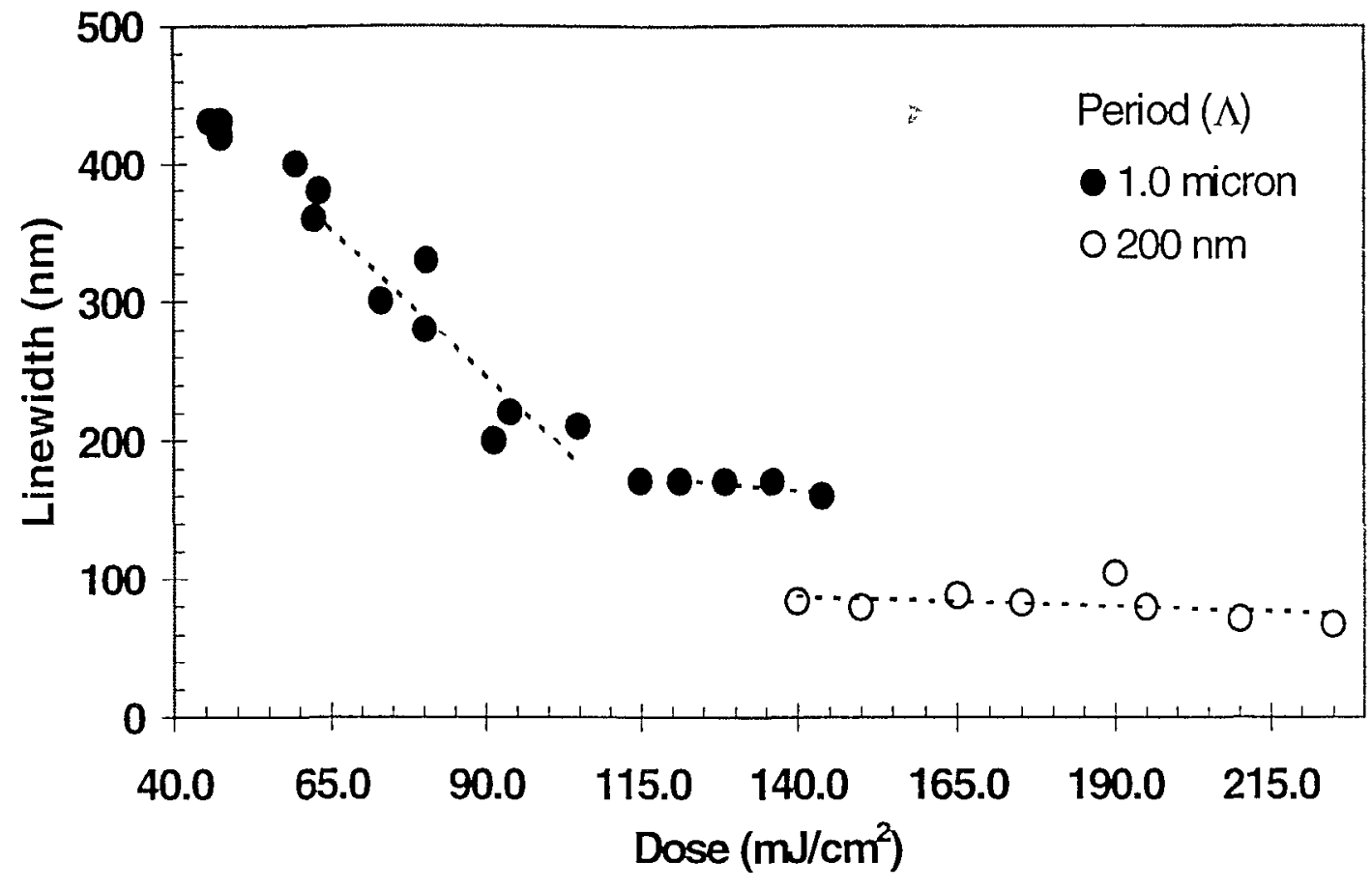

(b)

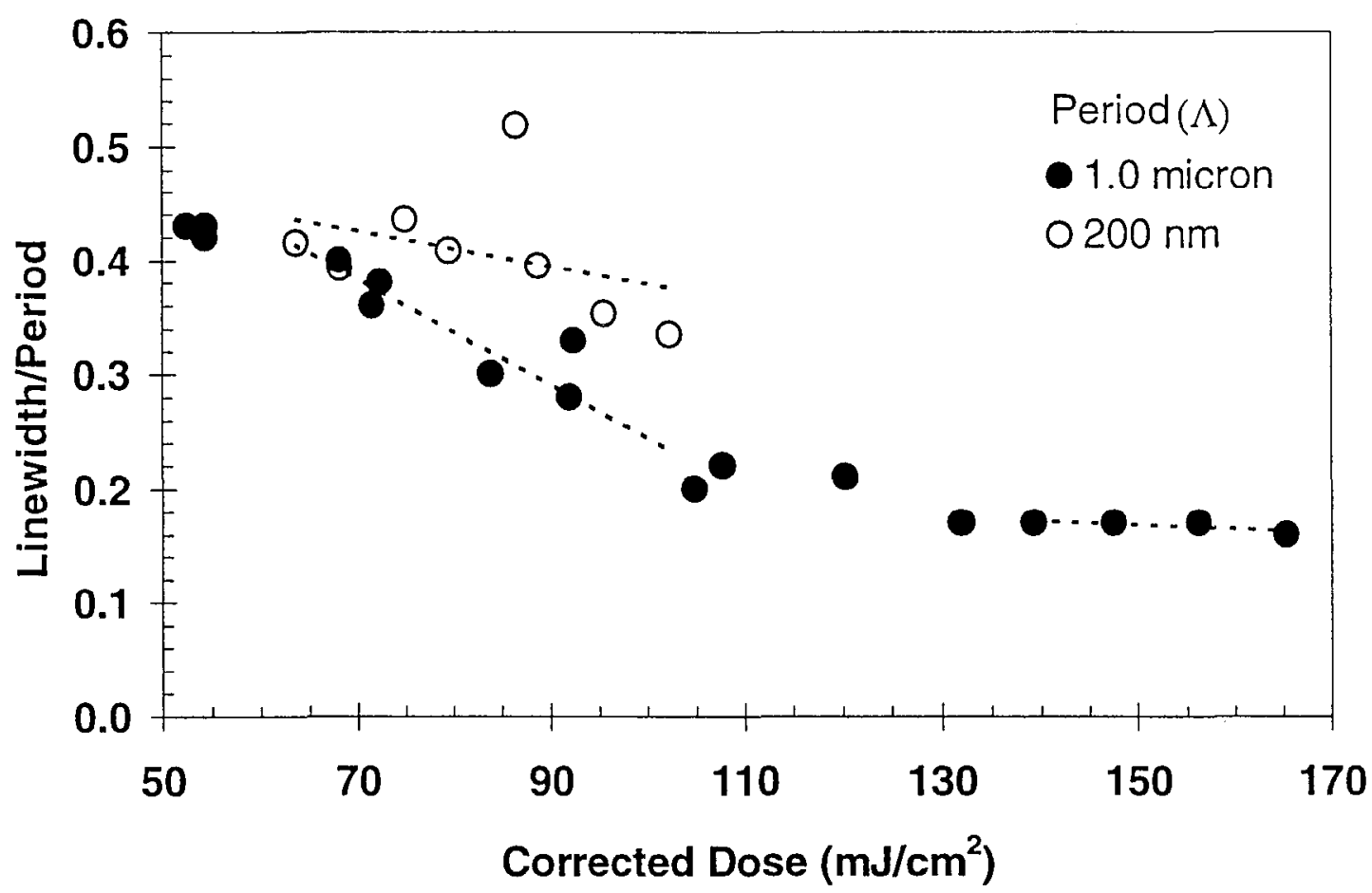

Figure 4: Results of linewidth measurements vs. exposure dose for $1.0-\mu \mathrm{m}$ and $200-\mathrm{nm}$ pitches. The dashed lines are linear fits using data points within the exposure range indicated. (a) Raw experimental data. Linewidths are measured at the base of each structure as indicated by the arrows in Figure 3(a). The dose is the sum of the measured fluence of both beams. (b) Normalized plot of experimental data. Linewidth is scaled by the period and exposure dose is corrected using the amplitude factors of Figure 5. The linear fits of each period in the dose range of $65-100 \mathrm{~mJ} / \mathrm{cm}^{2}$ have similar slopes suggesting that exposure latitude scales with interference period. 


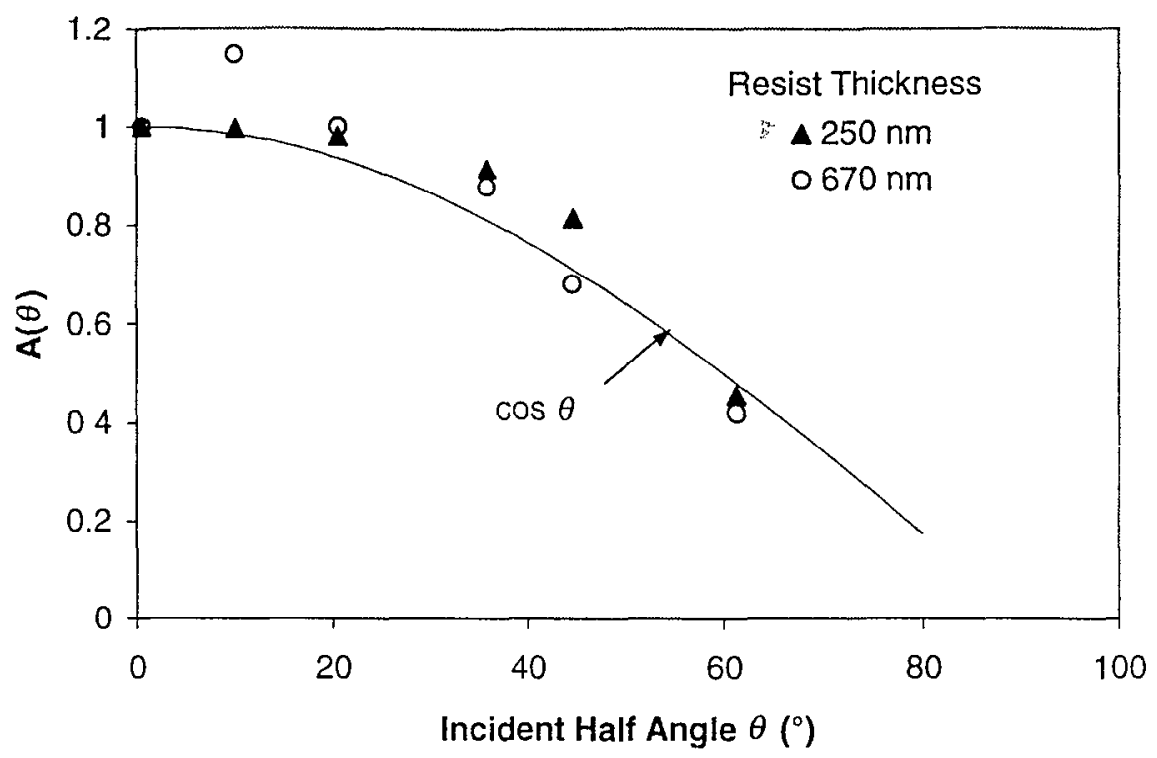

Figure 5: Plot of exposure dose correction factor vs. interference angle. The factors represent the relative amplitudes of the aerial image at the top of the resist and are calculated by PROLITH/2. They account for changes in reflectivity and standing wave effects for different resist thicknesses. Some $\cos \theta$ relationship is expected to account for the projected area in the exposure plane.

different resist thicknesses. There is some deviation from the predicted cosine trend indicating that the model also took into account effects associated with angular dependent reflectance and standing waves.

After normalizing the CD measurements by $\Lambda$ and the dose values by the appropriate correction factors given in Figure 5 , the data is plotted in Figure 4(b). It is evident from the figure that the curves for different $\Lambda$ do not overlay exactly, denoting that perfect scaling with interference period is not achieved For a given dose range, however, the normalized linear fits of both curves have similar slopes, indicating that exposure latitude does have some scaling relationship with period, at least to first order. Under the same premises, one can expect the exposure latitude for the 200 -nm period curve to eventually increase to a maximum value at larger doses, following the same general trend as the $1.0-\mu \mathrm{m}$ period data.

To further investigate scaling trends in IL, we configure PROLITH/2 to model our lithography process and generate simulated $C D$ vs. exposure dose curves. Necessary input parameters are first acquired through spectroscopic ellipsometry measurements ${ }^{23}$ of the refractive index $n$ and the extinction coefficient $k$ versus wavelength for the Shipley 507-A resist and ARC (see Table 1). We use Dill optical parameters for the resist at $\lambda=365 \mathrm{~nm}$. We also use dissolution parameters for Shipley MF-503, a developer with the same normality as MF-701. Figure 6(a) displays the simulated CD vs. dose curves of a $250-\mathrm{nm}$ thick resist layer for different values of $\Lambda$. The simulated curve for a $670-\mathrm{nm}$ thick layer at a $1.0-\mu \mathrm{m}$ pitch is also displayed.

TABLE 1. Refractive indices for resist and ARC

\begin{tabular}{lcc}
\hline \multicolumn{1}{c}{ Material } & \multicolumn{1}{c}{$\operatorname{lndex}(\lambda=351.1 \mathrm{~nm})$} \\
\hline Shipley 507-A & 1.70 & $k$ \\
Brewer Science ARC-XHRi-16 & 1.68 & 0.00 \\
\hline
\end{tabular}

Again, normalization of the axes of Figure 6(a) elucidates the scaling of exposure latitude with period. The CD is normalized by the period and the exposure dose is corrected with the appropriate factors in Figure 5. In the resulting plot, given in 

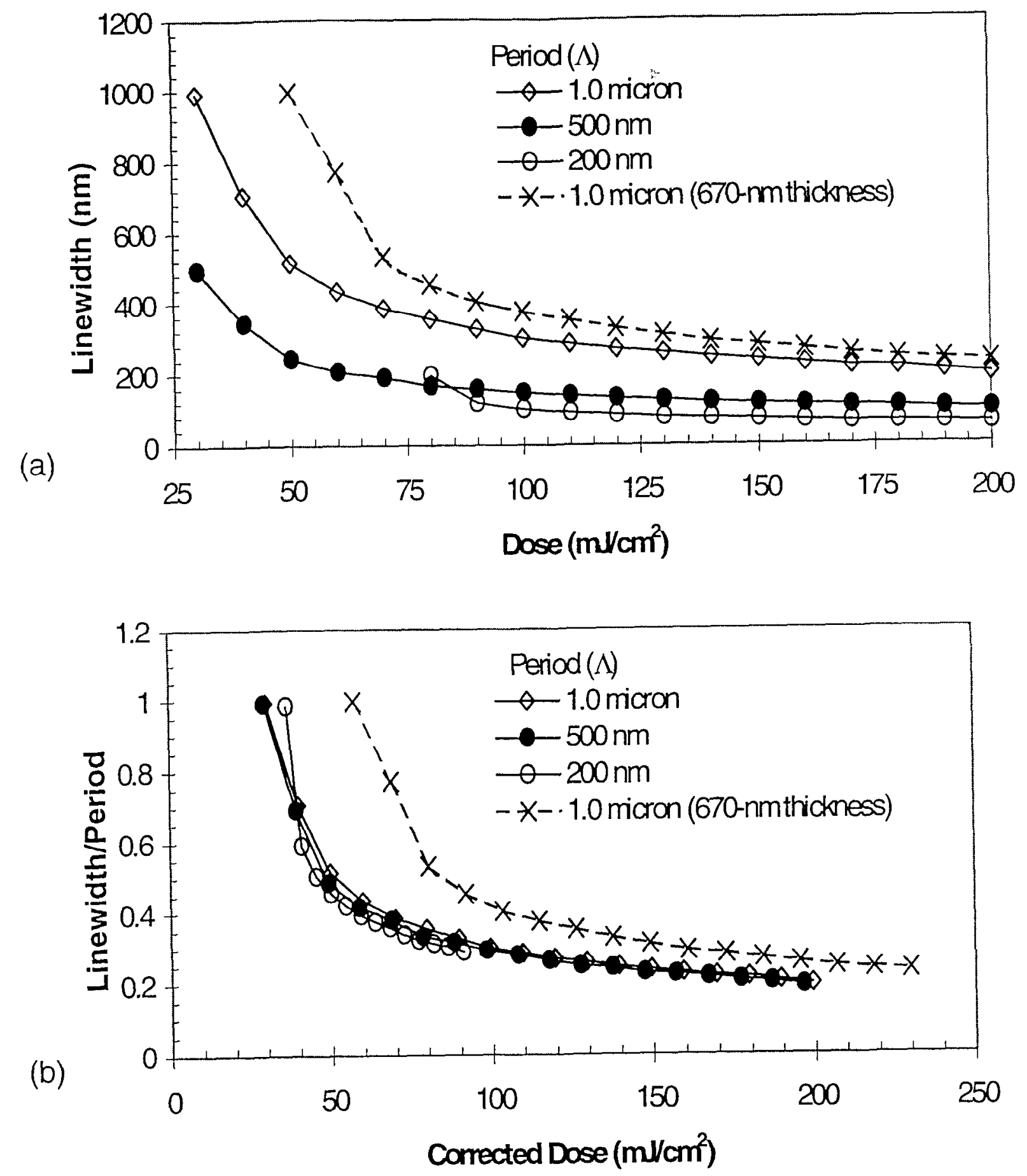

Figure 6: Simulated linewidth measurements vs. exposure dose for a 250-nm thick resist at different periods. A curve for a $670-\mathrm{nm}$ thick resist at a $1.0-\mu \mathrm{m}$ period is also shown. (a) Raw data before normalization (b) Normalized plot of simulated data. Linewidth is scaled by the period and dose is corrected by appropriate amplitude factors of Figure 5. Curves for 250-nm thickness are similar illustrating scaling behavior of exposure latitude with interference period. Some deviation occurs due to resist thickness. 
Figure 6(b), for $250-\mathrm{nm}$ thick resist, each curve has a similar shape and slope throughout the dose range suggesting that process latitude does scale with interference period.

Perfect scaling in IL can only be achieved in the limit of infinite resist contrast: This notion can be evidenced by use of C. A. Mack's Lumped Parameter Model (LPM) ${ }^{24}$ According to the LPM, the energy required to expose a feature width (CD) of $2 x$ in resist with contrast $\gamma$ can be determined by

$$
\varepsilon=\varepsilon_{0}-i(x)+\frac{1}{\gamma} \ln \left(\frac{d x}{d \varepsilon}\right)-\frac{1}{\gamma} \ln \left(\gamma_{d e v} r_{0}\right)
$$

where $\varepsilon, \varepsilon_{0}$ and $i(x)$ are the logarithms of the needed exposure energy $E$, dose-to-clear energy $E_{0}$ and the normalized aerial image intensity $I(x)$, respectively. Here, $t_{d e y}$ is the development time needed to just clear the photoresist for the energy $E_{0}$ and $r_{0}$ is the development rate. It has been assumed that the relative intensity variation with depth into the resist is constant. In the limit of very large $\gamma$, the last two terms of Equation (1) become negligible and the required energy is governed solely by the aerial image. In IL the normalized acrial image is a sinusoidal function given by

$$
I(x)=\frac{1}{2}\left[1+\cos \left(\frac{2 \pi x}{\Lambda}\right)\right]
$$

Transforming the width variable $x \rightarrow \zeta \Lambda$, where $\zeta$ is a dimensionless space variable, shows that this interference pattern scales laterally with period, i.e. $I(x) \rightarrow I(\zeta)$. Using Equations (1) and (2), it can then be shown that in the limit of infinite $\gamma$, the exposure latitude will also scale:

$$
\text { Exposure latitude } \propto \frac{d E}{E}=\frac{d I(\zeta)}{I(\zeta)}
$$

Equation (3) shows dependence only on the variable $\zeta$, therefore, if the $\mathrm{CD}$ is scaled by $\Lambda$ and the resist has infinite contrast, the exposure latitude at a specific scaled width will be the same for all $\Lambda$.

This scaling behavior has significant practical implications for IL. In terms of exposure, the processing of 80 -nm linewidths on a $200-\mathrm{nm}$ pitch is as straightforward as processing $170-\mathrm{nm}$ linewidths on a $1.0-\mu \mathrm{m}$ pitch, without degradation in resolution. To within certain limits, fabrication of a specific feature width with a wide degree of process latitude can be achieved, regardless of the size, provided that the optimal interference period is chosen. In addition, the largest process latitude does not necessarily occur at a 50\% duty cycle (1:1, linewidth : spacewidth), as shown by both our experimental data and the simulated curves (see Figures $4(\mathrm{~b})$ and $6(\mathrm{~b})$ ). It should be noted that different resists and processing conditions have different optimal duty cycles and periods, ${ }^{5}$ thus affording some flexibility and making IL an appealing technology.

The imperfect overlap of the curves in Figure $6(\mathrm{~b})$ suggests the presence of other effects that do not perfectly scale with interference period. At low doses $\left(<50 \mathrm{~mJ} / \mathrm{cm}^{2}\right)$, the seemingly random scatter of the data is primarily due to imprecision associated with numerically discrete calculations. At higher doses $\left(>50 \mathrm{~mJ} / \mathrm{cm}^{2}\right)$, each curve appears to have an offset which is monotonically related to the periodicity. This variation can be explained by considering the finite contrast of the resist in the context of the LPM. Performing the variable transformation $x \rightarrow \zeta \Lambda$ directly in Equation (1), the energy required to expose a feature width of $2 x$ becomes

$$
\varepsilon=\varepsilon_{0}-i(\zeta)+\frac{1}{\gamma} \ln (\Lambda)+\frac{1}{\gamma} \ln \left(\frac{d \zeta}{d \varepsilon} / \gamma_{d e v} r_{0}\right)
$$

Since $\gamma$ is now finite, the last two logarithmic terms are no longer negligible. It can be seen that the needed exposure energy now has a scaling relationship with $\Lambda$ modulated by a higher order, non-linear dependence associated with the resist's dissolution parameters. Consequently, the $C D$ vs. exposure dose curves of Figure 6(b) are similar in shape, but they are slightly shifted. In less ideal conditions than those governing Equation (4), there exists an intensity variation with depth into 
the resist. This variation contributes complicated absorption and standing wave effects that have been taken into account by the simulation, also resulting in deviation from perfect scaling behavior.

Although the simulations provide a comprehensive model of IL processing, some effects were not modeled, causing discrepancies between the simulated curves and the experimental data. The development parameters used in the simulations did not exactly describe our process. To accurately model a lithography system and provide predictive results, these dissolution constants must be precisely tuned to the specific process conditions, materials and equipment used. ${ }^{25-27}$ In the simulations performed, the beams were assumed to have equal intensities. Consequently, certain positions in the aerial image have zero-intensity nulls corresponding to portions of the resist that would never be exposed. In a more realistic system, the beam intensities may be mismatched, s-polarization may be imperfect, and the aerial image can suffer some degree of instability. If these modulations are time integrated, the effect is to add a constant bias to the entire aerial image. All regions of the resist become exposed, and with a large enough dose, no grating structure will remain. This effect is demonstrated in Figure $4(\mathrm{~b})$ by the gradual drop in $C D$ at the high-dose end of the $1.0-\mu \mathrm{m}$ data. In addition, the actual fluid dynamics of dissolution associated with reduced dimensions may contribute deviations between the simulated curves and the experimental data.

\section{SUMMARY}

To accommodate the high-resolution, high-throughput volume manufacturing needs of the progressing FPD industry, IL must provide adequate degrees of uniformity, repeatability and process control. We have shown that high-aspect ratio, steep sidewall 1-D gratings can be fabricated with wide exposure latitudes using IL. Because IL is a maskless technology, the aerial image generated to pattern gratings is not diffraction linited. Thus, the image scales with interference period, and fine linewidth control can be obtained for sub-quarter micron periods.

Using i-line technology, IL does not lack image resolution as does conventional lithography; nevertheless, process variables continue to affect uniformity, repeatability and CD-control capabilities. Both our experimental data and simulated resuits demonstrate that exposure latitude scales with interference period to first order. This scaling, however, is modulated by a non-linear dependence on period associated with processing parameters such as resist contrast and dissolution rate. In addition, the ordinary development of high-aspect ratio structures with periods on the order of $200 \mathrm{~nm}$ is complicated by rinse liquid surface tension. Thus, in order for IL to be a commercially viable alternative to conventional technology, these process limitations must be addressed.

\section{ACKNOWLEDGEMENT}

The authors would like to acknowledge helpful discussions with Mark L. Schattenburg and Chris A. Mack. This work was performed under the auspices of the U.S. Department of Energy by Lawrence Livermore National Laboratory under Contract W-7405-Eng-48.

\section{REFERENCES}

1. R. C. Sumner, "Microlithographic challenges for flat panel display production", Solid State Technol. 39, pp. 103-110, 1996.

2. T. A. Savas, M. L. Schattenburg, J. M. Carter, and H. I. Smith, "Large-area achromatic interferometric lithography for $100 \mathrm{~nm}$ period gratings and grids", J. Vac. Sci. Technol. B 14 (6), pp. 4167-4170, 1996.

3. R. D. Boyd, J. A. Britten, D. E. Decker, B. W. Shore, B. C. Stuart, M. D. Perry, and L. Li, "High-efficiency metallic diffraction gratings for laser applications", Appl. Opt. 34 (10), pp. 1697-1706, 1995.

4. M. D. Perry, R. D. Boyd, J. A. Britten, D. Decker, and B. W. Shore, "High-efficiency multilayer dielectric diffraction gratings", Opt. Lett. 20 (8), pp. 940-942, 1995.

5. M. L. Schattenburg R. J. Aucoin, and R. C. Fleming, "Optically matched trilevel resist process for nanostructure fabrication", J. Vac. Sci. Technol. B 13 (6), pp. 3007-3011, 1995.

6. E E. Scime, E. H. Anderson, D. J. McComas, and M. L. Schattenburg, "Extreme-ultraviolet polarization and filtering with gold transmission gratings", Appl. Opt. 34 (4), pp. 648-654, 1995.

7. A. Fernandez, H. T. Nguyen, J. A. Britten, R. D. Boyd, M.D. Perry, D. R. Kania, and A. M. Hawryluk, "Use of interference lithography to pattern arrays of submicron resist structures for field emission flat panel displays", J. Vac. Sci Technol. B 15 (3), pp. 729-735, 1997. 
8. J. P. Spallas, A. M. Hawryuk, and D. R. Kania, "Field emitter array mask patterning using laser interference lithography", J. Vac. Sci. Technol. B 13 (5), pp. 1973-1978, 1995.

9. X. Chen, S. H. Zaidi, and S. R. J Brueck, "Interferometric lithography of sub-micrometer sparse hole arrays for fieldemission display applications", J. Vac. Sci. Technol. B 14(5), pp. 3339-33 i9, 1996.

10. A. Fernandez, J. Y. Decker, S. M. Herman, D. W. Phillion, D. W. Sweeney, "Methods for fabricating arrays of holes using interference lithography", J. Vac. Sci. Technol. B 15 (6), pp. 2439-2443, 1997.

11. J. Y. Decker, A. Fernandez, D. W. Sweeney, "Generation of sub-quarter micron structures using optical interference lithography and image reversal", J. Vac. Sci. Technol. B 15 (6), pp. 1949-1953, 1997.

12. J. Nole, "Holographic lithography needs no mask", Laser Focus World 33 (5), pp. 209-212.

13. A. Fernandez, P. J. Bedrossian, SL. L. Baker, S. P. Vernon, and D R Kania, IEEE Trans. Magn. 32 (5), pp. 4472-4474, 1996.

14. S. H. Zaidi, S. R. J. Brueck, "Multiple-exposure interferometric lithography", J. Vac. Sci. Technol. B 11 (3), pp. 658-666, 1993.

15. T. Tanaka, M. Morigami, and N. Atoda, "Mechanism of resist pattern collapse during development process", Jpn. $J$ Appl. Phys. 32, pp. 6059-6064, 1993

16. H. Namatsu, K. Kurihara, M. Nagase, K. Iwadate, and K. Murase, "Dimensional limitations of silicon nanolines resulting from pattern distortion due to surface tension of rinse water", Appl. Phys. Lett. 66 (20), pp. 2655-2657, 1995.

17. Y. Yamashita, "Sub- $0.1 \mu \mathrm{m}$ patterning with high aspect ratio of 5 achicved by preventing pattern collapse", Jpn $J$ Appl. Phys. 35, pp. 2385-2386, 1996

18. T. Tanaka, M. Morigami, H. Oizumi, T. Ogawa, and S. Uchino, "Prevention of resist pattern collapse by flood exposure during rinse process", Jpn. J. Appl. Phys. 33, pp. L1803-L1805, 1994.

19. K. Deguchi, K. Miyoshi, T. Ishii and T. Matsuda, "Patterning characteristics of a chemically-amplified negative resist in synchrotron radiation lithography", Jpn J. Appl. Phys. 31, pp. 2954, 1992.

20. J Bauer, G. Drescher, and M. Illig, "Surface tension, adhesion and wetting of materials for photolithographic process", J. Vac. Sci. Technol. B 14 (4), pp. 2485-2492, 1996.

21 T Tanaka, M. Morigami, H. Oizumi, and T. Ogawa, "Freeze-drying process to avoid resist pattern collapse", Jpn J. Appl. Phys. 32, pp. 5813-5814, 1993.

22. FINLE Technologies, P.O. Box 162712, Austin, TX 78716.

23. Nanometrics Inc., 310 DeGuigne Drive, Sunnyvale, CA 94086.

24 C. A. Mack, Inside PROLITH: A Comprehensive Guide to Optical Lithography Simulation, Ch. 9, FINLE Technologies, Inc., Austin, 1997.

25. S.H. Thornton, C. A. Mack, "Lithography model tuning matching simulation to experiment", Optical Microlithography IX, G. E. Fuller ed., 2726, pp. 223-235, SPIE, Bellingham, WA, 1996.

26. K. P. Fahey, "Methods for measurement of development parameters in the manufacturing line for use in photolithography modeling", IEEE Trans. Semiconductor Manufacturing 9 (2), pp. 182-190.

27. M. E. Mason, R. A. Soper, R. M. Terry, and C. A. Mack, "Process-specific tuning of lithography simulation tools", Optical Microlithography X, Gene E Fuller, Ed., 3051, pp. 491-498, SPIE, Bellingham, WA, 1997. 


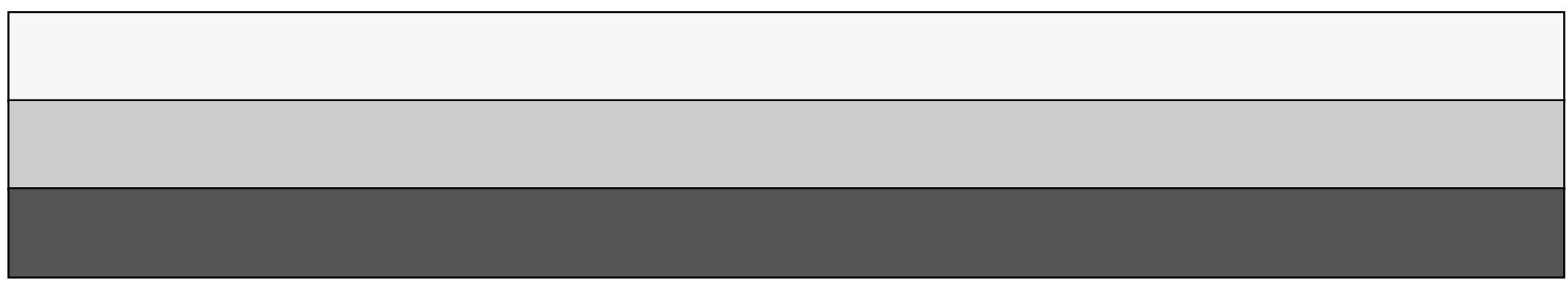

